\title{
Using a Quantum Dot as a High-Frequency Shot Noise Detector
}

\author{
E. Onac, ${ }^{*}$ F. Balestro ${ }^{\dagger}$ L. H. Willems van Beveren, U. Hartmann, ${ }^{\ddagger}$ Y. V. Nazarov, and L. P. Kouwenhoven \\ Kavli Institute of Nanoscience Delft, Delft University of Technology, P.O. Box 5046, 2600 GA Delft, The Netherlands
}

(Received 26 August 2005; published 1 May 2006)

\begin{abstract}
We present the experimental realization of a quantum dot (QD) operating as a high-frequency noise detector. Current fluctuations produced in a nearby quantum point contact (QPC) ionize the QD and induce transport through excited states. The resulting transient current through the QD represents our detector signal. We investigate its dependence on the QPC transmission and voltage bias. We observe and explain a quantum threshold feature and a saturation in the detector signal. This experimental and theoretical study is relevant in understanding the backaction of a QPC used as a charge detector.
\end{abstract}

PACS numbers: 72.70.+m, 73.21.La, 73.23.-b

On chip noise detection schemes, where device and detector are capacitively coupled within submillimeter length scales, can benefit from large frequency bandwidths. This results in a good sensitivity and allows one to study the quantum limit of noise, where an asymmetry can occur in the spectrum between positive and negative frequencies. The asymmetry, caused by the difference in the occurrence probability of emission and absorption processes, can be probed using quantum detectors [1]. In this Letter, we investigate the transport through a quantum dot (QD) under the influence of high-frequency irradiation generated by a nearby quantum point contact (QPC). The QPC current fluctuations induce photoionization, taking the QD out of Coulomb blockade, thereby allowing sequential tunneling through an excited state $[2,3]$. By studying this transient current while changing the QPC parameters, we show that we can perform high-frequency shot noise detection in the $20-250 \mathrm{GHz}$ frequency range.

The granularity of the electrons and the stochastic nature of their transport lead to unavoidable temporal fluctuations in the electrical current, i.e., shot noise [4]. For systems where transport is completely uncorrelated, such as vacuum diodes [5], noise is characterized by a Poissonian value of the power spectral density $S_{I}=$ $2 e I_{\mathrm{dc}}$. Here we use the QPC as a well-known noise source. When the QPC is driven out of equilibrium, i.e., by applying an electrochemical potential difference between the source and the drain of the QPC, a net current will flow if the QPC is not pinched off. At zero temperature $\left(k_{B} T \ll\right.$ $e V_{\mathrm{QPC}}$ ), the stream of incident electrons is noiseless, and shot noise, due to particle partition, dominates. The electrons are either transmitted or reflected, with a probability depending on the QPC transmission $T$. The power density can be written as $S_{I}=2 e I_{\mathrm{dc}} F$, where $F=\sum_{i=0}^{N} T_{i}(1-$ $\left.T_{i}\right) / \sum_{i=0}^{N} T_{i}$ is the Fano factor and the summation is over transport channels with transmissions $T_{i}$. In this case, correlations in the transport are introduced by the Pauli exclusion principle, resulting in a suppression of noise below the Poissonian value. Thus, shot noise vanishes if all the $1 D$ quantum channels either fully transmit $\left(T_{i}=1\right)$ or reflect $\left(T_{i}=0\right)$ [6].
In many recent experiments, QPCs are used as charge detectors [7]. In this context, our experiment provides information regarding the backaction [8-10] of the QPC when used as an electrometer for QD devices.

The QD and the QPC are defined in a GaAs/AlGaAs heterostructure, containing a two-dimensional electron gas (2DEG) at $90 \mathrm{~nm}$ below the surface, with an electron density $n_{s}=2.9 \times 10^{11} \mathrm{~cm}^{-2}$. We apply appropriate gate voltages such that we form a QD on the left and a QPC on the right [Fig. 1(a)]. The lithographic size of the QD is about $250 \times 250 \mathrm{~nm}^{2}$ and its charging energy, determined from standard Coulomb blockade measurements [Fig. 1(b)], is $E_{C}=1.3 \mathrm{meV}$. The QPC manifests conductance quantization [11] [Fig. 1(c)], understood in terms of the Landauer formula $G_{\mathrm{QPC}}=\left(2 e^{2} / h\right) \sum_{i=1}^{N} T_{i}$.

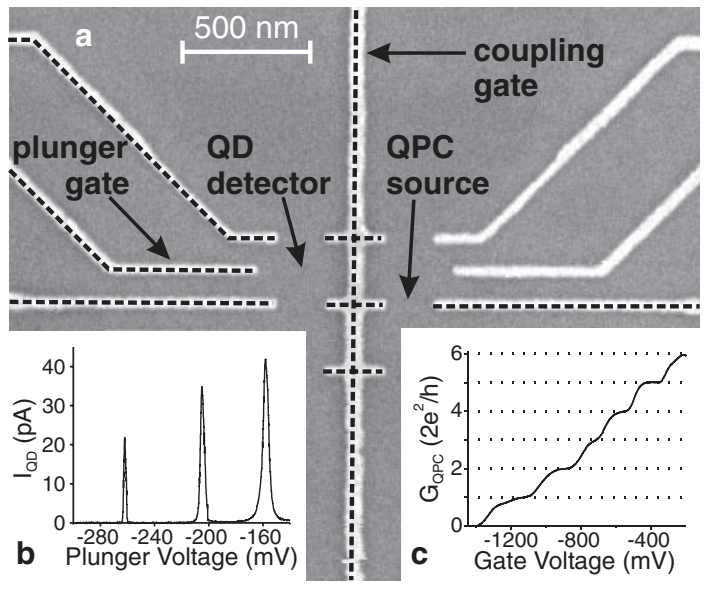

FIG. 1. (a) Scanning electron micrograph picture of the gate structure defined on top of the semiconductor heterostructure. The gates highlighted by dashed lines are used in the present experiment to define a QD on the left and a QPC on the right. All other gates are grounded. (b) QD current $I_{\mathrm{QD}}$ versus plunger gate voltage, for a voltage bias $V_{\mathrm{QD}}=30 \mu \mathrm{V}$, at $B=1.35 \mathrm{~T}$. (c) QPC conductance $G_{\mathrm{QPC}}$ as a function of the gate voltage at $B=0 \mathrm{~T}$. The potential applied to the coupling gate is kept constant. The QPC is used as a noise generator and the QD as a detector. 
We use the QPC as a noise generator that can be "switched" on or off by applying a voltage bias $V_{\mathrm{QPC}}$ and/or changing the QPC transmissions $T_{i}$. We measure transport through the $\mathrm{QD}$, as a function of the plunger gate voltage, under the influence of the QPC noise. A magnetic field perpendicular to the 2DEG can be used to adjust the transimpedance between the QPC and the QD [12]. Stray capacitances in the leads act as short circuits for highfrequency signals, and we use the impedance of edge states as an insulation between the source-detector part and the ground of the leads. In this way, the magnetic field enhances the coupling between the source and the detector. In this Letter, we present only measurements performed using the configuration in Fig. 1(a). Measurements with the opposite configuration, i.e., defining the QD on the right and the QPC on the left, have given identical results. Data were taken in a dilution refrigerator, with an effective electron temperature of $200 \mathrm{mK}$.

Our measurements are performed on a QD containing 10 electrons. This number was measured using the QPC as a charge detector for the QD [7]. The QD voltage bias $V_{\mathrm{QD}}=30 \mu \mathrm{V}$ is kept much smaller than the level spacing $(>200 \mu \mathrm{eV})$ between the ground state and the excited states of the QD. When the high-frequency noise generator is switched off (i.e., $V_{\mathrm{QPC}}=0$ or $T=\sum_{i} T_{i}$ has an integer value), we measure a single Coulomb peak in the current due to resonant tunneling through the ground state of the QD [see Fig. 1(b) or Fig. 2(a) for $T=0$ ]. In this situation, current can only flow through the QD when a charge state is positioned between the Fermi energies of the leads.

However, if the noise generator is switched on (i.e., when the QPC is set out of equilibrium by applying a bias voltage), additional current peaks emerge in the Coulomb blockade region. The amplitude of these peaks [labeled 1st es and 2nd es in Fig. 2(a)] depends on the QPC transmission and on the voltage applied to the QPC
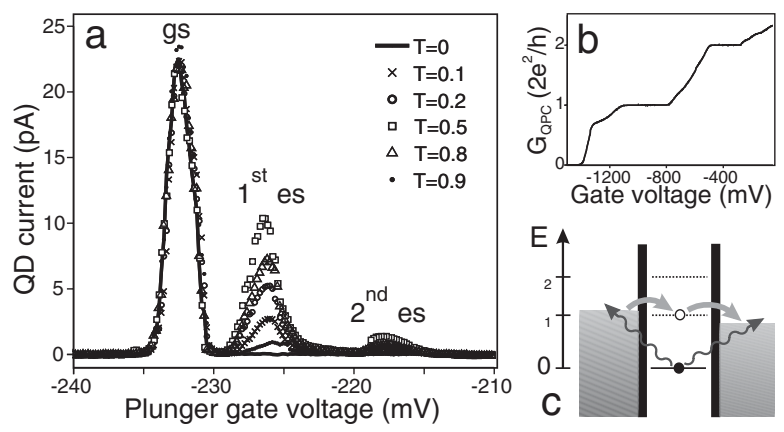

FIG. 2. (a) Current through the QD, as a function of the plunger gate voltage, under the influence of shot noise generated by the QPC. Measurements are performed at $B=1.35 \mathrm{~T}$, with $V_{\mathrm{QD}}=30 \mu \mathrm{V}$ and $V_{\mathrm{QPC}}=1.27 \mathrm{mV}$ and for different $\mathrm{QPC}$ transmissions. (b) QPC conductance versus the gate voltage at $B=1.35$ T. (c) Schematic representation of processes that lead to transport through the excited states of the QD.
[Fig. 4(a)]. Note that we also measure this effect when the QPC is current biased.

The additional peaks in the Coulomb blockade regime correspond, in energy, to the excited states of the QD. These energies are determined from spectroscopy measurements using large QD voltage bias. The energy levels of the 1 st and 2 nd excited states relative to the ground state [see Fig. 2(c)] are equal to $\varepsilon_{1}=245 \mu \mathrm{eV}$ and $\varepsilon_{2}=$ $580 \mu \mathrm{eV}$, respectively. The QPC gate voltage is adjusted during the QD measurement in order to compensate for the capacitive coupling of the plunger gate to the QPC. This allows us to have a fixed transmission $T$, while measuring the QD. We detect the current through the 1st excited state of the QD in dependence of the total QPC transmission from 0 to 2 and through the 2 nd excited state for $0<T<1$.

These data can be explained as follows. In the absence of noise, transport through the excited state is blocked since Coulomb blockade prevents having electrons in both the ground state and the excited state simultaneously. The appearance of transport peaks in the Coulomb blockade region is due to a photoionization process induced by the high-frequency shot noise generated by the QPC [13]. Here an electron in the ground state absorbs enough energy such that it can leave the dot to either one of the two leads. Subsequently, a transient current flows through the excited state, as long as the ground state stays empty [Fig. 2(c)]. This results in the appearance of conductance peaks, whenever an excited state is aligned between the Fermi levels of the leads. Current fluctuations through the QPC are thus converted into a dc current flowing through the excited state of the QD. This transient current can be analyzed in order to obtain information regarding the high-frequency fluctuations in the QPC.

For a theoretical description of our results, we first address the question of how the noise couples to the QD. The conversion of QPC current fluctuations into voltage fluctuations on the QD side is described by a circuit transimpedance [1] defined as $|Z(\omega)|=\sqrt{S_{V}(\omega) / S_{I}(\omega)}$, with $S_{I}(\omega)$ the current spectral density of noise generated by the QPC and $S_{V}(\omega)$ the power spectral density of voltage fluctuations at one barrier of the QD. This can be expressed as $|Z(\omega)| \approx|Z(0)|=\kappa R_{K}$, where $R_{K}=$ $e^{2} / h=25.8 \mathrm{k} \Omega$ is the quantum resistance and $\kappa$ is a dimensionless parameter describing the coupling between different QPC channels and QD barriers. In our model, we define four different $\kappa$ coefficients, depending on the channel involved in the QPC, and the barrier of the QD: $\kappa_{L, 1}$ and $\kappa_{L, 2}$ are the coupling coefficients between the first and the second channels, respectively, of the QPC and the left barrier of the QD, and $\kappa_{R, 1}$ and $\kappa_{R, 2}$ describe the coupling of the QPC channels to the right barrier. Experimentally, we can adjust the QD barriers in order to have symmetric escape rates to the left and the right reservoirs. The absence of pumping effects (i.e., regions where $I_{\mathrm{QD}}<0$ ) close to the Coulomb peaks [see Fig. 2(a)] 
indicates symmetric coupling for the QD barriers: $\kappa_{R}=$ $\kappa_{L}$. The only independent coupling parameters are $\kappa_{1} \neq$ $\kappa_{2}$ corresponding to the first two QPC channels. As already discussed, a perpendicular magnetic field can be used to increase the coupling parameter $\kappa$ (although this is not understood quantitatively).

The second question we address is what kind of energies and cutoff frequencies are involved in the photoionization process. In the low temperature limit, two energy scales are important for the detection mechanism. First, the energy difference $\varepsilon$ between the ground and the excited states of the QD is relevant, as the photoionization process pumps an electron out from the ground state. This level spacing [see Fig. 2(c)] sets a detector cutoff frequency $\nu_{\mathrm{QD}}=\varepsilon / h$, representing the minimum frequency that can induce photoionization [the minimum energy that can be detected, assuming single photon photon-assisted tunneling (PAT) processes]. The second relevant energy is provided by the QPC bias. This gives the cutoff frequency for the noise generator $\nu_{\mathrm{QPC}}=e V_{\mathrm{QPC}} / h$, corresponding to the maximum frequency that can be emitted (for independent tunneling events in the QPC). Thus, the frequencies contributing to the PAT process are in the range $\left[\nu_{\mathrm{QD}}, \nu_{\mathrm{QPC}}\right]$. For the measurements in Fig. 2(a), $V_{\mathrm{QPC}}=$ $1.27 \mathrm{mV}$, which corresponds to $\nu_{\mathrm{QPC}}=317 \mathrm{GHz}$, and, depending on the 1 st or 2 nd excited states, $\nu_{\mathrm{QD}}$ is equal to $\varepsilon_{1} / h=59 \mathrm{GHz}$ or $\varepsilon_{2} / h=140 \mathrm{GHz}$. These set two different detection bandwidths for the 1st and the 2 nd excited states, leading to different amplitudes for the detector signal (i.e., the peak height of the transient current).

Our model considers PAT in a QD. Noise generated by the QPC induces potential fluctuations between the QD energy levels and the electrochemical potentials in the leads. These fluctuations modify the tunneling rates $\Gamma_{L}$, $\Gamma_{R}$ between the QD and its source and drain leads. The change can be described using the theory of energy exchange with the environment [14]. If we consider the simple case of "classical" shot noise, where the power spectrum is white, the photoionization probability has a Lorenztian shape $P_{i}(E)=\left(1 / \pi w_{i}\right)\left[1 /\left(1+E^{2} / w_{i}^{2}\right)\right]$. The width $w_{i}=8 \pi^{2} \kappa_{i}^{2} T(1-T) e V_{\mathrm{QPC}}[1]$ includes the coupling coefficient as well as the classical noise power emitted by the QPC.

Using this theoretical model, we can fit the experimental results and obtain the parameters that characterize our system. We first extract the tunneling rate through the ground state of the QD by fitting the Coulomb peak when the noise generator is switched off (no additional peaks in the Coulomb blockade regime). We tune the system, by applying appropriate gate voltages on the electrodes, in order to have a symmetric QD: The two tunneling rates from QD to source $\left(\Gamma_{L}\right)$ and drain $\left(\Gamma_{R}\right)$ are equal. From the fit results, $\Gamma_{L}=\Gamma_{R}=0.575 \mathrm{GHz}$. The electron temperature and the voltage across the QD and across the QPC are known parameters and are, respectively, equal to
$200 \mathrm{mK}, 30 \mu \mathrm{V}$, and $1.27 \mathrm{mV}$. In order to explain the additional peaks in the Coulomb blockade regime, and the modulation of these peaks as a function of the QPC transmission, we introduce the following fit parameters: the escape rates $\Gamma_{1}^{\mathrm{es}}$ and $\Gamma_{2}^{\mathrm{es}}$ of the first and the second excited states and the coupling coefficients $\kappa_{1}$ and $\kappa_{2}$ to the first and the second channels of the QPC. By using this set of four fitting parameters, we are able to obtain a good fit for the QD current dependence on the plunger gate voltage, in the presence of noise (see inset in Fig. 3). The resulting fit values are reasonable: $\Gamma_{1}^{\mathrm{es}}=5.75 \mathrm{GHz}, \Gamma_{2}^{\mathrm{es}}=4.03 \mathrm{GHz}$, $\kappa_{1}=1.67 \times 10^{-2}, \kappa_{2}=4.83 \times 10^{-3}$. The coupling coefficients are difficult to estimate and they depend strongly on the details of the electromagnetic environment (e.g., on the geometry of the sample). The 1 order of magnitude difference between the coupling to the first and the second channels of the QPC is likely due to the shunting between the leads, provided by the first, conducting channel.

In Fig. 3, we plot the current flowing through the 1st (black square) and the 2nd (gray square) excited states of the QD as a function of the QPC transmission $T$. The points simply represent the current peak values as extracted from measurements presented in Fig. 2(a). We find that the QD detector signal is modulated by changing the QPC transmission in accordance to the shot noise theory: the noise vanishes for integer values $(T=1$ or $T=2)$ and is maximal for $T=0.5$ and close to $T=1.4$. The solid lines represent theoretical calculated values by making use of the previous determined parameters. We note that one set of fitting parameters can be used to describe the PAT signal dependence on both the QD energy and the QPC noise

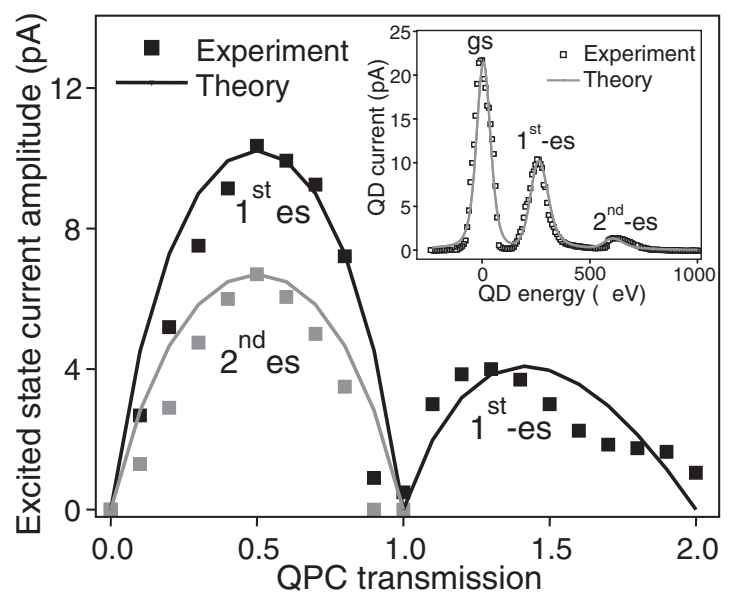

FIG. 3. Amplitude of the current through the excited state of the QD versus QPC transmission. Measurements are performed at $1.35 \mathrm{~T}$ with $V_{\mathrm{QD}}=30 \mu \mathrm{V}$ and $V_{\mathrm{QPC}}=1.27 \mathrm{mV}$. The current amplitude through the 2 nd excited state for $0<T<1$ and through the 1 st excited state for $1<T<2$ have been multiplied by a factor of 5 for clarity. Inset: QD current as a function of the QD energy for a QPC transmission $T=0.5$. Experimental points are in good agreement with the solid theoretical curve. The plunger gate value is converted in QD energy for clarity. 

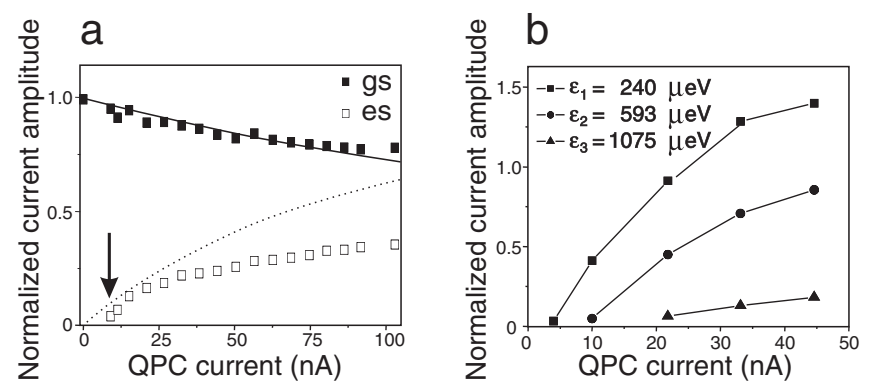

FIG. 4. (a) Normalized amplitude of current flowing through the ground and the excited states as a function of the QPC current. Measurements (presented as squares) are performed at $B=2.6 \mathrm{~T}$, with the QPC current biased at half transmission $T=0.5$. The solid and the dotted curves represent theoretical values obtained using parameters from the measurements at $B=$ 1.35 T. (b) Quantum limit cutoff frequencies corresponding to three excited states in the quantum dot detector. QPC transmission is set to $T \approx 1 / 7$.

power. In Fig. 3, a factor of 5 has been introduced in the vertical scaling for the 2 nd excited state and also for the 1 st excited state from $T=1$ to 2 , for clarity. The suppression of the detector signal for these two cases was already discussed: low amplitude of the 2nd excited state current is due to a smaller detection bandwidth, while the noise generated by the second QPC channel is partly screened by the electrons flowing through the first, ballistic channel. Screening is also the reason a QPC is less sensitive as an electrometer in the $T>1$ transmission range.

In Fig. 4(a), we measure and theoretically compute the saturation of the excited state current as a function of $I_{\mathrm{QPC}}$. The plot presents the current amplitude for the ground and the excited states, normalized to the amplitude of the Coulomb peak in the absence of noise. We clearly see that the amplitude of the excited state increases as a function of the QPC bias, while the amplitude of the ground state decreases. For the excited state, the difference between the experimental and the calculated amplitudes, at large QPC currents ( $>25 \mathrm{nA})$, is due to a second excited state and relaxation processes neglected in the theoretical calculation.

A distinct quantum feature present in the experimental measurements is the existence of a cutoff in the values of the QPC voltage bias [indicated by the arrow in Fig. 4(a)]. This corresponds to the condition $\nu_{\mathrm{QPC}}=\nu_{\mathrm{QD}}$ and represents the minimum QPC voltage bias for which the detection mechanism works. For smaller bias voltages, the emission side of the QPC noise is zero at the frequencies $\nu>\nu_{\mathrm{QD}}$ where the QD detector is sensitive. The theoretical results are obtained from a "classical," frequency independent expression for shot noise and, subsequently, they do not show this cutoff. Noise cutoff frequencies corresponding to three excited states are measured and presented in Fig. 4(b). At higher noise power, we measure a saturation for both amplitudes of current through the excited and the ground states. This phenomenon can be understood as reaching an equilibrium between PAT and QD relaxation processes.

In conclusion, we use a QD as an on chip quantum detector to achieve, for the first time, very high-frequency (in the range [20-250] GHz) shot noise measurements. We measure the cutoff, determined by the QPC bias, in the emission part of the noise spectrum. The detection process can also be viewed as a backaction of the QPC when used as a QD electrometer and represents an important source for the dark counts in the single-shot readout of individual electron spins in quantum dots [15].

We are grateful to R. Hanson for helping with the sample fabrication. We acknowledge technical assistance from R. Schouten and A. van der Enden. Financial support provided by the Dutch Foundation for Fundamental Research on Matter (FOM).

\footnotetext{
*Electronic address: eugen.onac@philips.com

†Present address: Laboratoire Louis Néel, associé au CNRS, B.P. 166, F-38042 Grenoble Cedex 9, France.

${ }^{\ddagger}$ Present address: Physics Department, ASC, and CeNS, Ludwig-Maximilians-Universität, D-80333 München, Germany.
}

[1] R. Aguado and L. P. Kouwenhoven, Phys. Rev. Lett. 84, 1986 (2000).

[2] T. H. Oosterkamp et al., Nature (London) 395, 873 (1998).

[3] T. Fujisawa, Y. Tokura, and Y. Hirayama, Phys. Rev. B 63, 081304(R) (2001).

[4] Y. M. Blanter and M. Büttiker, Phys. Rep. 336, 1 (2000).

[5] W. Schottky, Ann. Phys. (Leipzig) 57, 541 (1918).

[6] Y.P. Li et al., Appl. Phys. Lett. 57, 774 (1990); M. Reznikov, M. Heiblum, H. Shtrikman, and D. Mahalu, Phys. Rev. Lett. 75, 3340 (1995); A. Kumar et al., Phys. Rev. Lett. 76, 2778 (1996).

[7] M. Field et al., Phys. Rev. Lett. 70, 1311 (1993).

[8] S. A. Gurvitz, L. Fedichkin, D. Mozyrsky, and G. P. Berman, Phys. Rev. Lett. 91, 066801 (2003).

[9] E. Buks et al., Nature (London) 391, 871 (1998).

[10] M. Avinun-Kalish et al., Phys. Rev. Lett. 92, 156801 (2004).

[11] B. J. van Wees et al., Phys. Rev. Lett. 60, 848 (1988).

[12] The precise magnetic field values were chosen corresponding to minima in the Shubnikov-de Haas oscillations in the bulk 2DEG. This implies that no backscattering occurs and that the incident stream of electrons is noiseless [e.g., see M. Henny et al., Science 284, 296 (1999)].

[13] T. H. Oosterkamp et al., Phys. Rev. Lett. 78, 1536 (1997).

[14] G. L. Ingold and Y. V. Nazarov, in Single Charge Tunneling, edited by H. Grabert and M.H. Devoret (Plenum, New York, 1992).

[15] J. M. Elzerman et al., Nature (London) 430, 431 (2004). 